\title{
Relations between pulmonary venous branches and bronchial and arterial branches
}

Discipline of anatomy, Department I - preclinical disciplines, Faculty of medicine, University "Ovidius" Constanţa

\begin{abstract}
Our results were obtained on 24 cases, 12 plastic molds and 12 dissections, examining the relations between the branches of origin of the pulmonary veins, and of their roots and trunks with the corresponding bronchuses and arteries.

We assessed the situation of the superior and inferior right pulmonary veins, of the superior, middle and inferior lobes pedicles, together with the general assessment of the right pulmonary pedicles. Same is on the left. We cannot speak of a general sample that applies to the broncho-pulmonary vascular pedicles or to the relations between the pulmonary veins and their branches with broncho-arterial elements, as they are quite varied; they are characteristic of each lung lobe and even segment, being unable to assess standard relations only in the hilum. When variation in number occurs, even into the hilum we may have altered relations. Also is quite common that vascular branches may pass from one lobe to another, as long as the pulmonary fissures are rarely complete.
\end{abstract}

Keywords: pulmonary veins, relations, bronchus, artery

\section{Dr. Iliescu D.M.}

Department of Anatomy, Faculty of medicine, University "Ovidius" Constanţa, Romania

Aleea Universitatii, Nr. 1, Campus B

Constanța, Romania

dan@anatomie.ro

\section{Introduction}

The venous blood, brought to the lungs through the two pulmonary arteries, is returned to the heart as arterial blood through the pulmonary veins, vessels with converging branches, usually two veins for each lung $[1,2,3,4,5,6,7,8,9]$. They arise from the capillary networks of the lobules and last bronchial branches, forming larger vessels up to the lung hilum, were they form three trunks for the right lung and two trunks for the left, so a trunk for each lobe. The trunk originating from the right superior lobe joins the trunk of the middle lobe, so that the right lung has two pulmonary veins such as the left one $[1,2,7]$. Whatever their size, the pulmonary veins satellite the bronchial branches like the arterial branches, placing preferably opposite to the corresponding artery. Some may pass isolated, without any regular relation with either bronchial or pulmonary branches $[1,2]$. Approaching the lung hilum, they are placed anterior to the arterial branches, which are located anterior to the bronchi [1.2].

According to [3 and 5], within the lung parenchyma, the pulmonary veins have an independent traject, as they move towards the pulmonary hilum. According to [6], the superior right pulmonary vein, that drains oxygenated blood from the upper and middle lobes, is formed by two roots that join one another in front of the pedicle; this trunk, with variable length, crosses at right angle the anterior face of the 
pulmonary artery, passing obliquely infero-medial and posterior. Also [6] shows that the inferior right pulmonary vein drains the inferior lobe by two roots (upper and lower), forming a short, horizontal trunk, that passes slightly posterior and superior towards the left atrium. The same author [6] describes the left superior pulmonary vein formed by the confluence of three branches while the left inferior pulmonary vein is formed by the confluence of the two intersegmental branches and leaves out of the lung hilum at its posterior part, substantially below the other components of the pedicle, with a transverse direction slightly upward [6]. According to [9], the branches of the pulmonary veins are separated from the bronchial because they between the pulmonary segments and drain blood from one segment (intrasegmental portion) or even two neighboring segments (intersegmental portion). Therefore, the arterial and venous branches should be named differently [9].

\section{Material and Methods}

Our study was performed on 24 cases, of which 12 were dissected samples and other 12 were plastic casts. Plastic casts resulted from Technovit 7143 injection in fresh lungs, followed by corrosion with $\mathrm{NaOH}$. We assessed the relations between the tributaries of the pulmonary veins and of their roots and trunks with the corresponding arteries and bronchuses.

\section{Results}

The superior right pulmonary vein is formed of two roots, the upper and lower, frequently occurring on the side of the division of the right artery and the right bronchus. The upper root passes anterior to the inferior and middle lobar arteries and anterior to the inferior lobar bronchus, to join the upper arterial branch while the lower root, usually located posterior to the anterior one, is disposed anterior to the lower lobar artery and corresponding lower bronchus.

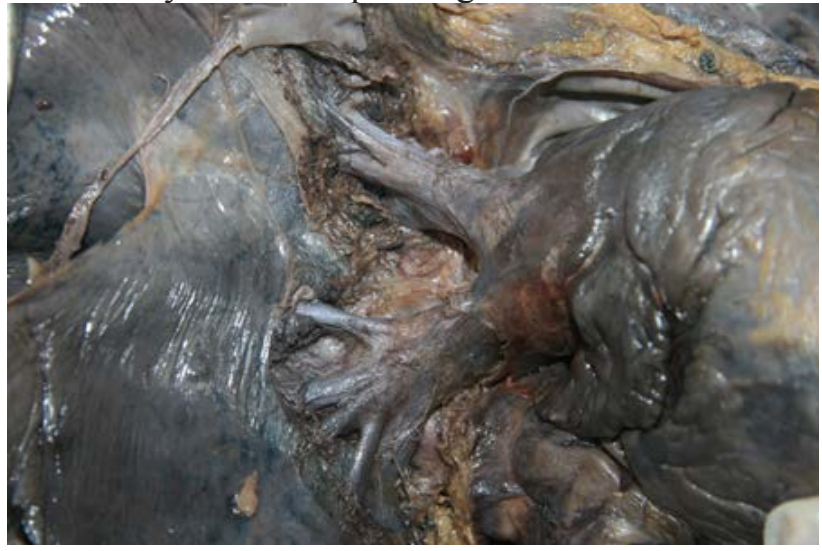

Figure 1 - Anterior view of lung. Superior pulmonary vein is located anterior-inferior superior root of the upper bronchus root is located above the lower lobar bronchus medium lung. Lower right pulmonary vein is located anterior-inferior lobar bronchus and end in the lower right atrium above and below it has superior pulmonary lung. Apical lower lobe vein rami in his trajectory to the trunk vein passes anterior inferior lobar bronchus branches basal vein is located anterior-inferior lobar bronchus inferior.

The inferior right pulmonary vein formed of two roots, upper and lower, the superior formed usually by two branches, superior and inferior, initially situated posterior to the pedicular elements and towards the side of the lung to be situated anterior to the branches of the bronchi but remaining posterior to the arterial branches. The inferior root branches are placed posterior to the arterial branches.

The pedicle of the superior lobe shows the following arrangement: anterior the vein, artery in the middle and the bronchus is posterior. Sometimes, the arrangement of the different elements of the upper lobe is different within the lower half with respect to their disposal in the upper half. Into the lower half the elements are: antero-lateral is the vein, posteromedial to the vein is the superior lobar artery and postero-lateral to the artery is the superior lobar bronchus while into the upper half of the superior lobe the available items are: antero-lateral is the 
vein, postero-medial to the vein is the bronchus and posterior to the bronchus is the artery.

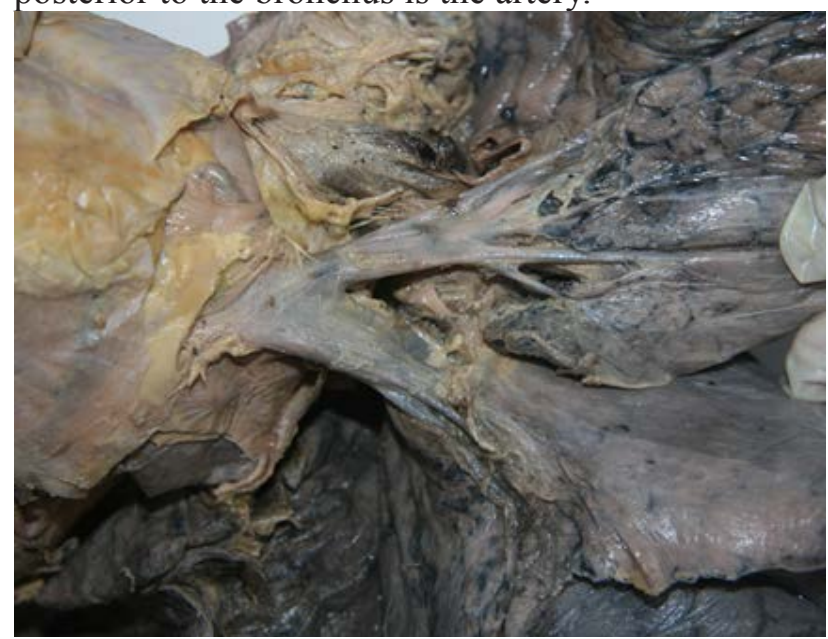

Figure 2 - Anterior view of left lung. Superior pulmonary vein is located on a plane above the bottom. Superior pulmonary vein superior root more voluminous as the inferior, is located anterior superior bronchial artery and proper, and lower root strictly lingular is formed from two upper and lower branches flanking the artery and bronchus. Forming vein is medial superior pulmonary bronchial branching. Inferior pulmonary vein is located anterior-inferior broncho-arterial elements

Into the middle lobe, the arrangement of the pedicle elements, from anterior to posterior, is: anterior is the vein (first root), in the middle is the artery and posterior is the bronchus. Sometimes branches ending in the inferior right pulmonary vein drain the middle lobe. Alternatively, the middle pedicular elements are: the artery is higher, in the middle the bronchus and inferior the vein.

Anteriorly, in the apical part of the inferior lobe, the vein is located anterior to the bronchus and the artery and, within the postero-superior pedicle, the arrangement is: the artery posterior, the bronchus is anterior-inferior to it and the vein is postero-inferior,

Within the middle part of the inferior lobe, the arrangement of the pedicle, from superior to inferior, is: artery, bronchus and vein (slightly posterior to the bronchus). In the antero-inferior part of the inferior lobe are, frequently, two pedicles; one is superior and showed the following arrangement: anterior is the artery and posterior are, within the same plane, the vein superior and the bronchus inferior. The second pedicle, the inferior one, presented the artery anterosuperior, the bronchus infero-medially and the vein posterior.

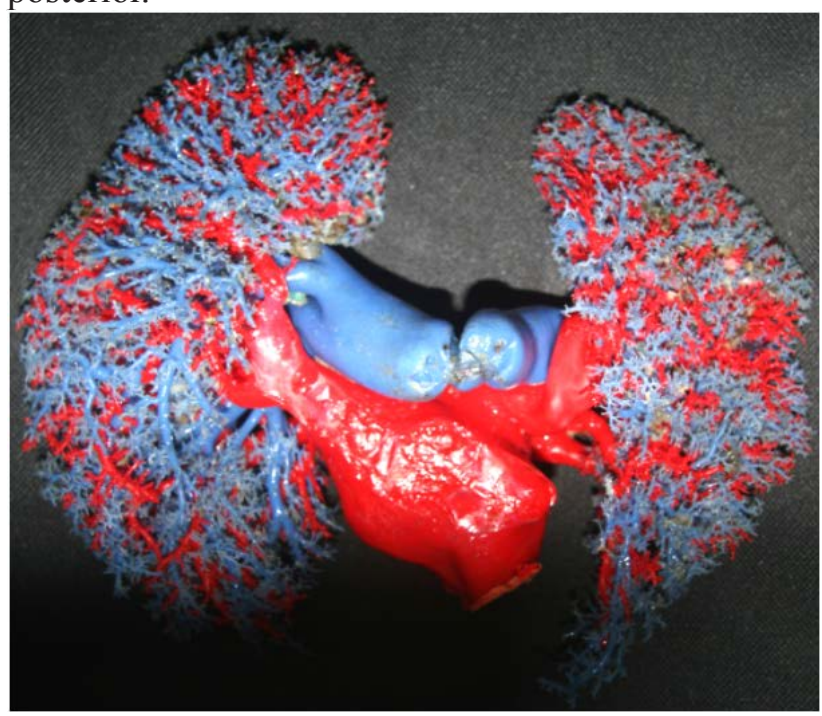

Figure 3 - Anterior view injection of the veins and arteries only. Right upper pulmonary vein is located anterior-superior to bottom, which partially masks (is vein above). Subsequently, the upper right pulmonary vein trunk is straight and has lower pulmonary artery flush with it, ending in the right atrium $1.2 \mathrm{~cm}$ above the lower right vein and its on a higher plane $0.6 \mathrm{~cm}$. The root of the upper three arms forming the posterior venous path. Lower root is oblique infero-medial direction, forming the upper root angle 280. Is arranged in a plane slightly posterior to superior root, located above arterial branches. Anterior lower root is crossed by an oblique infero-lateral ram arterial origin middle lobar artery, which descends to the supero-lateral part of the basal portion of the lower lobe. Posterior inferior lobar artery is crossed. Left upper pulmonary vein in a plane posterior ends $(0.4 \mathrm{~cm})$ and lower $(1 \mathrm{~cm})$ right upper pulmonary vein. It arranged $1.5 \mathrm{~cm}$ from the upper left and lower

it to $0.3 \mathrm{~cm}$ distance between them being $3.5 \mathrm{~cm}$. Is formed from two roots that confluiază flush with the left pulmonary artery branch disposed posterior vein. After confluence, the trunk oblique infero-medial vein down the front of the left pulmonary artery and on the underside of a curve its supero-medial and posterior passes inferomedial face of the left pulmonary artery located above it, to finish left supero-lateral right atrium. The upper root is disposed above the artery, with which it forms a vascular only on the rear face of the lobe, the front arms showing various arterio-venous thin. Lower root is arranged on a plane slightly above the upper root. And the basic level on the front can not speak of the vascular pedicle 
Towards the lateral border of the right inferior lobe, the arrangement of the broncho-vascular elements is highly variable, the venous branches being located posteriorly (most commonly) or anteriorly to the arterial and bronchial branches. In the inferior lobe, on its posterior face, there are thinner venous branches without an arterial or bronchial correspondent.

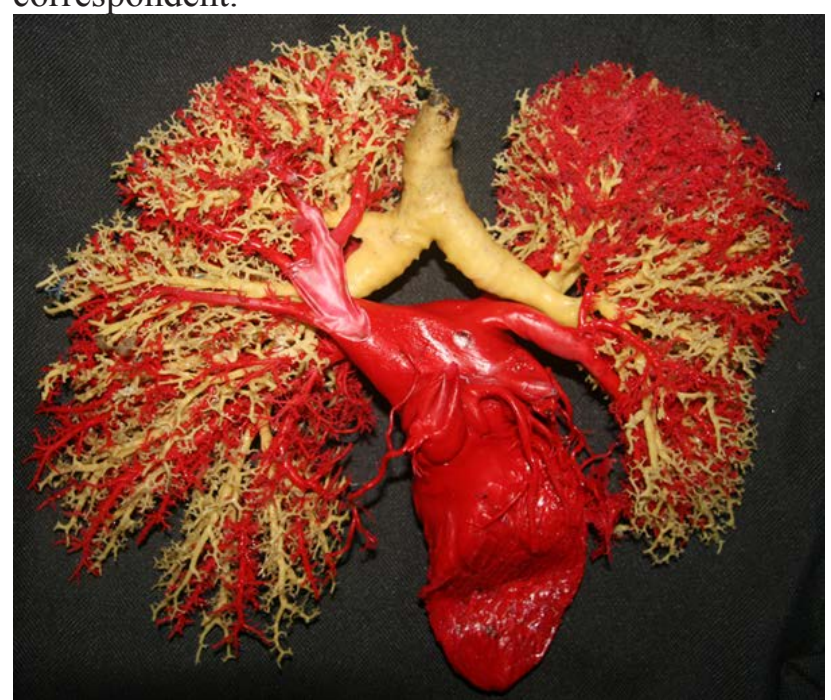

Figure 4 - Anterior view. Injection of trachea and pulmonary veins. Right upper pulmonary vein (above) shows the upper root located medially located anterior rami bronchial properly being disposed below the lower root bronchial rami. Middle lobar vein is formed of two branches, anterior and posterior branches being stacked and arranged slightly above bronchial the middle branch. Left pulmonary vein before (superior) is formed from two roots, above (bigger) and posterior vena $1.2 \mathrm{~cm}$ terminating at the posterior and inferior vena anterior by $0.3 \mathrm{~cm}$ from it. Its branches are located above the corresponding bronchial branches. In other segments, basal bronchial branches are located above the vein.

Within the right hilum, the arrangement of the pedicular elements on its anterior face is: the artery anterior, the vein in the middle and the bronchus posterior to it. In some cases the superior pulmonary vein is located antero-inferior, the inferior lobar branch of the pulmonary artery is postero-superior to the vein and the right main bronchus is posterosuperior to the artery. Sometimes, on the posterior face of the right hilum, the arrangement of the elements is: slightly posterior and inferior is the vein, antero-superior is the bronchus and antero-inferior is the inferior lobar arterial branch.

The superior left pulmonary vein is formed medial to the termination of the artery and left bronchus. It is formed of two roots, upper and lower, both passing anterior to the left posterior pulmonary vein. The superior root passes posterior to the superior lobar bronchus and anterior to the left pulmonary artery. The inferior root, located postero-lateral to the superior one, passes anterior to the left inferior lobar vein. Initially it is placed below and slightly anterior to the lingular bronchus, and then inferior to the lower bronchus (and in the same plane), with the lingular artery posterior to the bronchus.

The inferior left pulmonary vein has the intrapulmonary venous branches anterior to the other items, frequently being disposed posterior to the bronchial branches.

Within the superior lobe the arterial branches are located anterior to the venous. In the middle part of the upper lobe, the venous branch can be located antero-inferior to the bronchial one, which in turn is located antero-inferior to the arterial. Into the apical segment, in the anterior part the vein is located medial to the artery and in the posterior part the venous branches are placed anterior the other elements, with the arterial branches located posterior to the bronchi.

At lingular level, the arterial branches are located posterior to the venous but anterior to the bronchi. Most commonly, all segmental pedicles of the lower lobe have the same arrangement of elements: superior is the artery, in the middle (postero-inferior to the artery) the bronchus and inferior and slightly posterior to the bronchus is the vein. Sometimes, on the anterior face of the basal region of the inferior lobe, the elements are, from anterior to posterior: vein, artery, bronchus.

The arrangement in the left pulmonary hilum is: anterior the pulmonary artery, in the middle (posterior and slightly superior) the bronchus and postero-inferior the vein.

On the anterior face, at the level of the hilum, the left vein is located inferior and anterior to the left pulmonary artery and the venous branches are arranged above the arterial and bronchial ones. On the posterior face, at the level of the hilum, the pulmonary vein is inferior and slightly anterior to the bronchus 
and the artery is anterior to the left bronchus.

\section{Discussion}

Usually, the pulmonary vessels, venous and arterial, regardless of their caliber, accompany the bronchial branches, forming the vascular-nervous pulmonary pedicles, in which the arrangement is characteristic of each lung and its structural unit (lobe, segment). The most common branches of the vein are located opposite the arteries [1,2]. More rarely, we encountered cases where venous branches independent of the route of bronchial artery or proper or even independently of the other two components of the pedicle, variant signaled by [1.2]. Bouchet [6] classified the elements of the right upper lobe pedicle into two groups, mediastinal and fissure. Mediastinal group the most posterior is upper lobar bronchus (upper lobar bronchus is easily accessible by the posterior mediastinal pleura incision), before it is a plan arterial (upper lobe mediastinal arteries), and the above is mediastinal venous trunk, forming a range of venous branches which converge towards the front of the pedicle by crossing at right angles to the front face of the bronchi and blood components, thus hindering a more or less prior to the access thereof. The same upper lobar pedicle elements I met and new specification that sometimes superior lobe bronchovascular elements may alter the provision in the lower half of the lobe in relation to their disposal in the upper half of the lobe. Thus, the upper half of the upper lobe of available elements is anterolateral vein, posterior medial vein bronchus and bronchus posterior artery, and the lower half of the upper lobe pedicle is formed by: antero-lateral vein, posterior medial vein artery upper lobar and lobar bronchus artery posterolateral upper bronchus is one that can change position, fissure-like layout elements.

After [6] pedicle lobe medium theory is best individualized because the elements are grouped. We have encountered cases where the middle lobe vein is vascularized by venous branches ending in the lower right pulmonary vein, variant in which the disposal pedicular elements are middle lobar artery superior, middle bronchus and inferior vena .

After [6] the right lower lobe pedicles are relatively simple, inferior pulmonary vein is located on the postero-inferior pedicle right lung, bronchus is the most profound, being masked lateral of the arterial main trunk,after giving one or two arteries apical still has a short posterior lateral trajectory face bronchus, giving birth to the basal arteries. We have found that the root superior pulmonary vein inferior posterior bronchus initially located in the lower lobe lateral segments disposal bronchovascular elements is highly variable, venous branches can be frequently located posterior and anterior to the bronchi and blood. [6] found that the left superior pulmonary vein, which drains than upper lobe is located at the anterior-inferior pedicle being approached about mediastinum. Superior lobe receives venous pedicle veins originating from the lingula and the efferent branches the benchmark plan cleavage [6], variant I encountered frequently. Lingular arterial pedicle is easy to spot elements in the back of fissure. Their dissection allows to easily discover lingular bronchus. We have found that the root of the left superior pulmonary vein superior in its path to the confluence with the lower root passes posterior superior lobar bronchus and pulmonary artery above the left. Lower root, situated on the upper level posterolateral initially is located anterior-inferior bronchus lingular, and later to have lower bronchus and in the same plane, artery lingular being located posterior to bronchus.

The elements of left lower lobe pedicle is quite similar to the existing right side [6]. The left lower lobe of the apical segment has venous drainage by interapicobasal veins which should be sectioned, representing the reference numeral of the extirpation of the cleavage plane [6]. Left superior pulmonary vein, which drains than upper lobe is located at the anterior-inferior part of the pedicle [6]. We have encountered cases in which lingular vein ends and a ramus of the apical lobe of lower left lobe. Generally all pedicles lower lobe segmentations have the same disposition of elements: superior artery, middle (posterior inferior artery) and slightly lower bronchus and bronchus rear, ram vein. I have encountered cases where the disposal items anterior inferior lobe 
basal area, from anterior to posterior, can be: vein, artery, bronchus. There are cases where the back of the lower lobe can not speak of broncho-vascular pedicle only showing trunks artery well represented, sending branches posterior-anterior to the front of the left lower lobe and cases in which the front face not broncho-vascular pedicle are only showing vein, venous trunks represented, sending branches anterior-posterior to the back of the left lower lobe, a point which I found quoted in the literature consulted. For [10] the disposal is above the pulmonary lung root and for inferior is back back disposal. For [8] in their extrapericardic portion pulmonary veins are pre bronhial and the inferior pulmonary veins are infra bronchial. We have found that the right lung hilum frequently artery, the vein being located anterior and bronchus is disposed posterior to veins. Sometimes superior pulmonary vein is located anterior inferior artery and right main bronchus is superior posterior artery. In the left lung hilum is located above the left pulmonary artery superior pulmonary vein, left bronchus being located above and posterior artery.

\section{Conclusions}

We can not speak of a general provision applies to elements broncho-pulmonary vascular pedicle, relations pulmonary veins and their branches with broncho-arterial elements, because they are very different, being characteristic of each lobe and right lung segment, unable to establish relationships standard than the lung hilus, the branches bronchovascular large, but at this level relationship may change when there are many variants of one of the pedicle elements. Are quite common that vascular branches may switch from one lobe to another, as pulmonary fissure rarely complete, aspect mentioned by [6]. Most commonly, the superior pulmonary vein blood can collect the apical segment of the lower lobe of the lung. Also frequently ram upper middle lobe vein (as the lung) or lingular segment (the lung left) ends the root superior pulmonary vein superior and not inferior root, as stated [3].

Since frequently the two veins of a lung parenchymal not comply territory drained, upper or lower, may participate in both territories vasculature in these cases and could name them front and rear, relative to their position at the completion of the left atrium.

\section{Refrences}

1. Testut, L. (1924). Livre III. Appareil de la respiration et de la phonation. (pp. 832-838). Paris: Ed. Gastoin Doin

2. Testut, L. (1921). Traité d'anatomie humaine. Angeiologie. (pp. 265-266). Paris: Ed. Gastoin Doin

3. Rouvière H. \& Delmas A. (1997). Anatomie Humaine. Descriptive, topographique et fonctionelle, Tome 2. (pp. 305-306). Paris: Ed. Masson

4. Bourgeon A. \& Chevrel J.P. (1994). Anatomie clinique. Le Tronc-Les poumons. (pp. 219-240). Paris: Ed. Springer-Verlag

5. Moore, K., Dalley, A. \& Agur, A.M. (2012). Anatomie clinică. Fundamente și aplicaţii. (pp. 116). București: Ed. Callisto

6. Bouchet, A. \& Cuilleret, J. (1991). Anatomie topographique, descriptive et fonctionnelle. Le cou. Le torax. (pp. 1067-1076, 1117-1122, 11361140). Paris: Ed. Simep

7. Standring S. (2005) Gray's Anatomy. The Anatomical Basis of Clinical Practice. (pp. 1027). Ed. Elsevier-Churchill Livingstone

8. Kamina, P. (1997). Anatomie. Vol. 11. Dos et Thorax. (pp. 175-178). Paris: Ed. Maloine.

9. Schunke, M., Schulte, E., Schumacher, U., Voll, M. \& Wesker, K. (2005). Atlas d'Anatomie. Cou et organes internes. (pp. 120-121). Paris: Ed. Maloine

10. Beauthier, J.P. \& Lefevre, Ph. (1993). Traté d'Anatomie. De la théorie à la pratique palpatoire. Tome 3. Tête et tronc. Propédeutique 
viscérale. (pp. 248). Bruxelles: Ed. De Boeck Université

11. $* * * * *$ Federative Committee on Anatomical Terminology. (1988). Terminologia Anatomica. International Anatomical Terminology. (pp. 9192). Stuttgart: Thieme Verlag 